\title{
Cetuximab could be more effective without prior bevacizumab treatment in metastatic colorectal cancer patients
}

This article was published in the following Dove Press journal:

OncoTargets and Therapy

II November 2015

Number of times this article has been viewed

Yasuyoshi Sato

Satoshi Matsusaka

Mitsukuni Suenaga

Eiji Shinozaki

Nobuyuki Mizunuma

Department of Gastroenterology, Cancer Institute Hospital, Japanese Foundation for Cancer Research,

Tokyo, Japan
Correspondence: Satoshi Matsusaka Department of Gastroenterology, Cancer Institute Hospital, Japanese Foundation for Cancer Research, 3-8-3I Ariake, Koto-ku, Tokyo I35-8550, Japan

$\mathrm{Tel}+8 \mathrm{I} 3352001 \mathrm{II}$

Fax $+8|33520014|$

Email satoshi.matsusaka@jfcr.or.jp
Background: Cetuximab and bevacizumab reportedly improve the survival of patients with metastatic colorectal cancer (mCRC), but their most effective sequence of administration is unknown. The aim of this study was to compare the survival of patients with mCRC treated with cetuximab after bevacizumab failure with that of patients with $\mathrm{mCRC}$ without previous bevacizumab therapy.

Patients and methods: In total, 190 of 323 patients with mCRC treated with cetuximab from March 2006 to July 2013 were enrolled in our hospital for this retrospective study. Forty-seven patients were treated with cetuximab-based second-line therapy, 21 of whom had received prior bevacizumab; 143 patients were treated with cetuximab-based third-line therapy, 109 of whom had received prior bevacizumab. The Kaplan-Meier method with a log-rank test and Cox regression analysis were performed to evaluate the overall survival and progression-free survival (PFS) of each group of patients.

Results: The median follow-up time was 11.8 months in patients who received second-line cetuximab-based chemotherapy and 13.7 months in those who received third-line cetuximabbased chemotherapy. Univariate analysis revealed that the median PFS was significantly longer in patients without prior bevacizumab therapy than in patients with prior bevacizumab therapy (second line, $P=0.048$; third line, $P=0.0022$ ). Multivariate analysis adjusted for baseline characteristics showed that third-line cetuximab-based chemotherapy with or without prior bevacizumab was significantly associated with PFS $(P=0.014)$. Neither the presence nor the absence of prior bevacizumab administration was associated with overall survival.

Conclusion: Cetuximab could be more effective without prior bevacizumab. Prior bevacizumab use may decrease the efficacy of cetuximab.

Keywords: cetuximab, bevacizumab, EGFR, VEGF, metastatic colorectal cancer

\section{Introduction}

Addition of cetuximab or bevacizumab to chemotherapy has been shown to improve the overall survival (OS) of patients with metastatic colorectal cancer (mCRC). However, the efficacy of cetuximab when administered after bevacizumab failure remains unknown.

In the Phase III FIRE-3 trial conducted by the German AIO CRC Study Group, the addition of cetuximab to FOLFIRI as first-line treatment of KRAS wild-type (exon 2 [codon 12/13]) mCRC provided a 3.7-month increase in OS compared with the addition of bevacizumab. However, progression-free survival (PFS) was not improved. ${ }^{1}$

The Phase II PEAK study of 285 treatment-naive patients with KRAS wild-type mCRC compared panitumumab with bevacizumab + mFOLFOX6. Two hundred seventy-eight patients received treatment. The median PFS was 10.9 months with 
panitumumab and 10.1 months with bevacizumab (hazard ratio [HR], 0.87; 95\% confidence interval [CI], 0.65-1.17; $P=0.35$ ). The median OS was 34.23 months with panitumumab and 24.3 months with bevacizumab (HR, 0.62; 95\% CI, 0.44-0.89; $P=0.009)$. These results indicate that there is no significant difference between anti-epidermal growth factor receptor (anti-EGFR) therapy and anti-vascular endothelial growth factor (anti-VEGF) therapy as first-line therapy for KRAS wild-type mCRC. ${ }^{2}$ This trend is similar to that in the FIRE-3 study from the viewpoint of comparison of anti-EGFR drugs with bevacizumab.

A retrospective study of 58 patients by Norguet et al indicated that previous anti-VEGF therapy decreases cetuximab efficiency. ${ }^{3}$ This result indicates that sequential use of cetuximab after bevacizumab failure may decrease the efficacy of cetuximab, which could explain the results of the FIRE-3 trial.

Additional retrospective analysis of the FIRE-3 trial indicated that second-line application of antibodies was favored in patients with shorter first-line PFS, suggesting that preplanned second-line therapy may not reflect therapeutic reality. Accordingly, second-line treatment without antibodies was associated with longer OS than was antibodybased treatment. A strong trend toward a shorter OS was observed in patients receiving cetuximab as second-line therapy after bevacizumab. Although the backgrounds of the patients undergoing second-line therapy were unknown and may have affected the outcomes, the trend toward a shorter OS in patients receiving cetuximab as second-line treatment after bevacizumab was remarkable. ${ }^{1}$

Based on this prior evidence, sequential use of anti-EGFR therapy after failure of anti-VEGF therapy might decrease the efficacy of anti-EGFR therapy. Whether the sequential use of cetuximab after bevacizumab failure decreases the efficacy of cetuximab remains unknown. The aim of our analysis was to evaluate the outcome of sequential treatment with cetuximab-based chemotherapy after bevacizumab failure in patients with $\mathrm{mCRC}$.

\section{Patients and methods Study eligibility}

This study included 190 patients with KRAS wild-type mCRC treated with cetuximab-based chemotherapy after bevacizumab failure from March 2006 to July 2013 in our hospital. Forty-seven patients were treated with cetuximab as second-line treatment; 21 of these patients had been previously treated with bevacizumab. One hundred fortythree patients were treated with cetuximab as third-line treatment; 109 of these patients had been previously treated with bevacizumab (Figure 1). OS was defined as the duration of time from the start of cetuximab therapy to death of any cause. PFS was defined as the duration of time from the

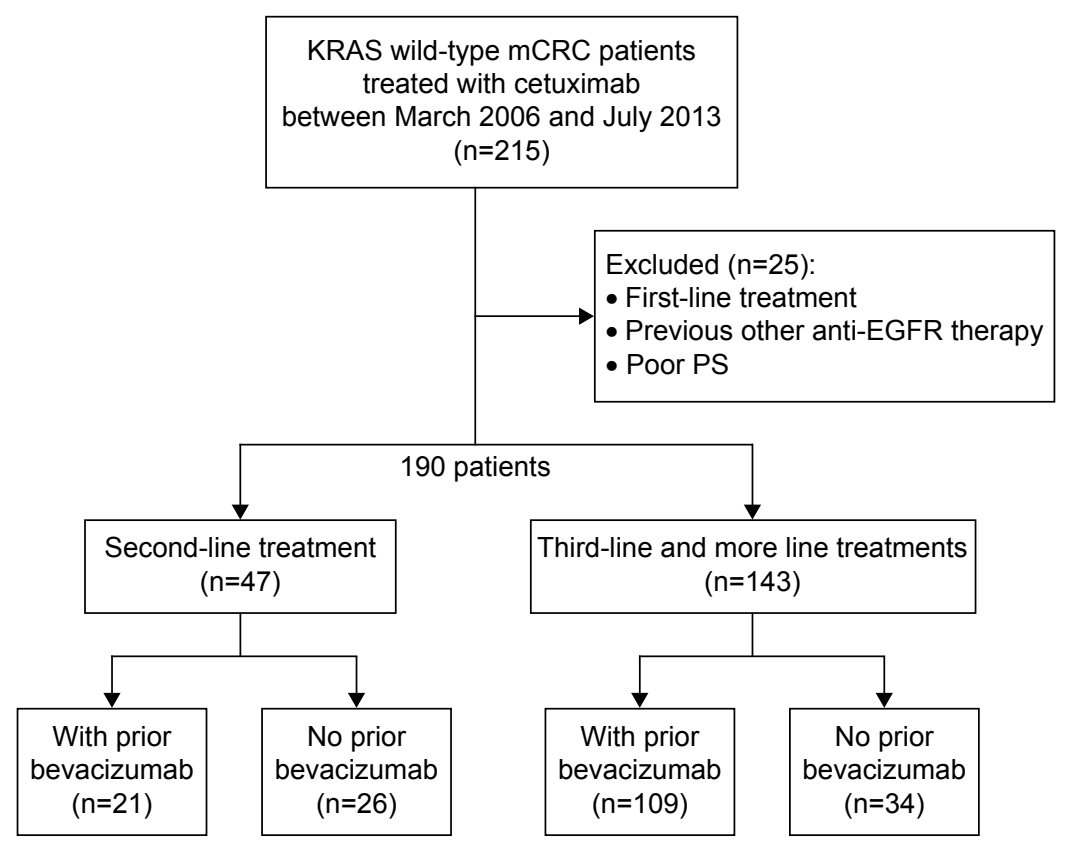

Figure I One hundred and ninety patients with KRAS wild-type mCRC treated with cetuximab-based chemotherapy after bevacizumab failure between March 2006 and July 2013 were enrolled in our hospital.

Notes: Forty-seven patients were treated with cetuximab as second-line treatment; 21 of these patients had been previously treated with bevacizumab. One hundred and forty-three patients were treated with cetuximab as third-line treatment; 109 of these patients had been previously treated with bevacizumab.

Abbreviations: mCRC, metastatic colorectal cancer; PS, performance status; EGFR, epidermal growth factor receptor. 
start of cetuximab therapy to the first recorded occurrence of oncologist-assessed disease progression. OS after first-line treatment was defined as the duration of time from the start of the first-line treatment to death of any cause.

\section{Statistical analysis}

We estimated the OS and PFS using the Kaplan-Meier method stratified by prior bevacizumab use. The patients were divided into the no prior bevacizumab use group and the prior bevacizumab use group. Univariate analysis between clinical factors and OS or PFS was performed with the logrank test. Cox proportional hazards models were used for multivariate analysis of clinical factors with $P$-values $<0.2$ in the univariate analyses. A $P$-value $<0.05$ was considered to indicate a statistically significant difference in the multivariate analysis.

All statistical analyses were performed with $\mathrm{R}$ (The $\mathrm{R}$ Foundation for Statistical Computing). This study received ethics approval from the Scientific Review Board and Institutional Review Board of Cancer Institute Hospital, Japanese Foundation for Cancer Research. Written, informed consent was obtained from all patients.

\section{Results}

\section{Patient characteristics}

Comparison of the two patient groups showed that the no prior bevacizumab use group had a greater number of patients who had undergone second- and third-line therapies with age $\geq 65$ years, male sex, Eastern Cooperative Oncology Group (ECOG) performance status (PS) $\geq 1$, and unresectable primary tumors (Tables 1 and 2). The prior bevacizumab use group had a greater number of patients who had undergone second- and third-line FOLFIRI + cetuximab therapies (Tables 1 and 2). The reasons for the lack of bevacizumab administration in the no prior bevacizumab use group included uncontrolled hypertension, a history of ischemic heart disease, a history of cerebral infarction, active bleeding or a high risk of bleeding, abscess, protracted wound healing, thromboembolism or tumor embolism, proteinuria, old age, and others (Table 3). EGFR expressions by immunohistochemistry were examined in 98 patients (52\%; Table 4).

\section{Statistical analysis in second-line cetuximab group}

The median follow-up time was 11.8 months (range, 0.5947.6 months) in the second-line cetuximab group. The median OS was 8.98 months $(95 \% \mathrm{CI}, 4.89-26.2)$ in the no prior
Table I Patients' characteristics in cetuximab as second line

\begin{tabular}{|c|c|c|}
\hline Characteristics & $\begin{array}{l}\text { With prior } \\
\text { bevacizumab } \\
(n=21)\end{array}$ & $\begin{array}{l}\text { Without prior } \\
\text { bevacizumab } \\
(n=26)\end{array}$ \\
\hline \multicolumn{3}{|l|}{ Age } \\
\hline Median (yr) & 58.4 & 63.6 \\
\hline Range (yr) & $31-75$ & $36-81$ \\
\hline$>65 \mathrm{yr}, \mathrm{n}(\%)$ & $5(23)$ & $13(50)$ \\
\hline \multicolumn{3}{|l|}{ Sex, n (\%) } \\
\hline Male & $14(67)$ & $18(69)$ \\
\hline Female & $7(33)^{\prime}$ & $8(3 I)$ \\
\hline \multicolumn{3}{|l|}{ ECOG PS, n (\%) } \\
\hline 0 & $16(76)$ & $16(62)$ \\
\hline I & $4(19)$ & $9(35)$ \\
\hline 2 & I (5) & I (4) \\
\hline \multicolumn{3}{|l|}{ Primary site, n (\%) } \\
\hline Cecum & $0(0)$ & $0(0)$ \\
\hline Vermix & $0(0)$ & $2(8)$ \\
\hline Ascending colon & $4(19)$ & I (4) \\
\hline Transverse colon & $\mathrm{I}(5)$ & I (4) \\
\hline Descending colon & I (5) & $2(8)$ \\
\hline Sigmoid colon & $7(33)$ & $9(35)$ \\
\hline Rectum & $8(38)$ & $11(42)$ \\
\hline Nonresection of primary, $\mathrm{n}(\%)$ & $5(24)$ & $9(35)$ \\
\hline \multicolumn{3}{|l|}{ Metastatic sites, n (\%) } \\
\hline Liver & $12(57)$ & $14(53)$ \\
\hline Lung & $4(19)^{\prime}$ & $14(53)$ \\
\hline Lymph node & $13(62)$ & $12(46)$ \\
\hline Peritoneal dissemination & $8(38)$ & $7(27)^{\prime}$ \\
\hline Bone & $2(10)$ & $2(8)$ \\
\hline Brain & $0(0)$ & $0(0)$ \\
\hline Others & $2(10)$ & $5(19)$ \\
\hline \multicolumn{3}{|l|}{ Regimen, n (\%) } \\
\hline Cetuximab monotherapy & $4(19)$ & $8(31)$ \\
\hline CPT-II + cetuximab & $3(14)$ & $6(23)$ \\
\hline FOLFIRI + cetuximab & $14(67)$ & $12(46)$ \\
\hline
\end{tabular}

Abbreviations: PS, performance status; yr, years; ECOG, Eastern Cooperative Oncology Group.

bevacizumab use group and 7.72 months (95\% CI, 3.95-8.91) in the prior bevacizumab use group ( $P=0.117$; Figure $2 \mathrm{~A})$. In the no prior bevacizumab use group, the analysis showed a favorable trend but no significant difference in OS. The median PFS was 4.44 months (95\% CI, 2.43-13.3) in the no prior bevacizumab use group and 3.09 months $(95 \% \mathrm{CI}$, $1.81-4.14)$ in the prior bevacizumab use group $(P=0.048)$. In the no prior bevacizumab use group, the analysis showed a significant difference in PFS (Figure 2B). Univariate analysis for OS showed that the clinical factors with $P$-values $<0.2$ were prior bevacizumab use, PS $\geq 1$, sex, lung metastasis, and lymphatic metastasis. Multivariate analysis showed that lymphatic metastasis was associated with OS ( $P=0.0011$; HR, 2.93; 95\% CI, 1.53-5.59). Univariate analysis for PFS showed that the clinical factors with $P$-values $<0.2$ were age $\geq 65$ years, prior bevacizumab use, $P S \geq 1$, liver metastasis, lung metastasis, and lymphatic metastasis. Multivariate analysis showed that age $\geq 65$ years $(P=0.049$; HR, 0.33 ; 95\% CI, 0.16-0.72), PS $\geq 1$ ( $P=0.034$; HR, 2.27; 95\% CI, 
Table 2 Patients' characteristics in cetuximab as third line

\begin{tabular}{|c|c|c|}
\hline Characteristics & $\begin{array}{l}\text { With prior } \\
\text { bevacizumab } \\
(n=109)\end{array}$ & $\begin{array}{l}\text { Without prior } \\
\text { bevacizumab } \\
(n=34)\end{array}$ \\
\hline \multicolumn{3}{|l|}{ Age } \\
\hline Median (yr) & 59.4 & 64.0 \\
\hline Range (yr) & $28-79$ & $38-77$ \\
\hline$>65$ yr, n (\%) & $35(32)$ & $20(59)$ \\
\hline \multicolumn{3}{|l|}{ Sex, n (\%) } \\
\hline Male & $50(46)$ & $25(74)$ \\
\hline Female & $59(54)$ & $9(26)$ \\
\hline \multicolumn{3}{|l|}{ ECOG PS, n (\%) } \\
\hline 0 & $90(83)$ & $22(64)$ \\
\hline 1 & $18(16)$ & $12(35)$ \\
\hline 2 & $\mathrm{I}(\mathrm{I})$ & $0(0)$ \\
\hline \multicolumn{3}{|l|}{ Primary site, n (\%) } \\
\hline Cecum & $2(2)$ & $0(0)$ \\
\hline Vermix & I (I) & $0(0)$ \\
\hline Ascending colon & $12(11)$ & $4(12)$ \\
\hline Transverse colon & $8(7)$ & $3(9)$ \\
\hline Descending colon & $5(5)$ & I (3) \\
\hline Sigmoid colon & $38(35)$ & $12(35)$ \\
\hline Rectum & $43(39)$ & $14(4 \mid)$ \\
\hline Nonresection of primary, $\mathrm{n}(\%)$ & $17(16)$ & $10(29)$ \\
\hline \multicolumn{3}{|l|}{ Metastatic sites, n (\%) } \\
\hline Liver & $72(66)$ & $26(76)$ \\
\hline Lung & $72(66)$ & $24(7 I)$ \\
\hline Lymph node & $54(50)$ & $24(7 I)$ \\
\hline Peritoneal dissemination & $32(29)$ & $6(18)$ \\
\hline Bone & $7(6)$ & $2(6)$ \\
\hline Brain & $0(0)$ & $0(0)$ \\
\hline Others & $14(13)$ & $9(26)$ \\
\hline \multicolumn{3}{|l|}{ Regimen, n (\%) } \\
\hline Cetuximab monotherapy & $14(13)$ & $5(15)$ \\
\hline CPT-II + cetuximab & $92(84)$ & $28(82)$ \\
\hline FOLFIRI + cetuximab & $3(3)$ & I (3) \\
\hline
\end{tabular}

Abbreviations: PS, performance status; yr, years; ECOG, Eastern Cooperative Oncology Group.

1.06-4.84), and lymphatic metastasis ( $P=0.0037$; HR, 3.00; 95\% CI, 1.42-6.31) were associated with PFS.

\section{Statistical analysis of third-line cetuximab group}

The median follow-up time was 13.7 months (range, 0.85-55.0 months) in the third-line cetuximab group. Eighty-seven ( $80 \%$ ) of 109 patients with prior bevacizumab use had received bevacizumab beyond progression. The median OS was 16.1 months (95\% CI, 9.6-18.0) in the no prior bevacizumab use group and 12.2 months $(95 \% \mathrm{CI}$, 9.3-15.5) in the prior bevacizumab use group $(P=0.22)$ (Figure 3A). In the no prior bevacizumab use group, the analysis showed a favorable trend but no significant difference in OS. The median PFS was 8.35 months $(95 \%$ CI, 6.35-10.4) in the no prior bevacizumab use group and
Table 3 The reasons why bevacizumab was not administered in the no prior bevacizumab use group

\begin{tabular}{lll}
\hline Reason, $\mathbf{n}(\%)$ & Second line & Third line \\
\hline Before approval of bevacizumab & I (2) & $6(17)$ \\
Hypertension & $2(4)$ & $6(17)$ \\
History of ischemic heart disease & $0(0)$ & $2(6)$ \\
History of cerebral infarction & I (0) & $4(12)$ \\
Bleeding/easily bleeding & $5(I I)$ & $3(9)$ \\
Abscess & $0(0)$ & $3(9)$ \\
Protracted wound healing & $0(0)$ & $2(6)$ \\
Thromboembolism or tumor embolism & I (2) & $2(6)$ \\
High risk of perforation & $6(12)$ & $0(0)$ \\
Uric protein positive & I (2) & $0(0)$ \\
Cavity-forming lung lesion & $0(0)$ & I (3) \\
Old age & $4(8)$ & $3(9)$ \\
Unknown & I (2) & $3(9)$ \\
\hline
\end{tabular}

4.80 months (95\% CI, 4.04-5.50) in the prior bevacizumab use group ( $P=0.0022$ ). In the no prior bevacizumab use group, the analysis showed a significant difference in PFS (Figure 3B). In univariate analysis for OS, clinical factors with $P$-values $<0.2$ were PS $\geq 1$ and liver metastasis. Multivariate analysis showed that $\mathrm{PS} \geq 1(P=0.0017$; HR, 1.94; 95\% CI, 1.30-3.07) was associated with OS. In univariate analysis for PFS, clinical factors with $P$-values $<0.2$ were prior bevacizumab use, male sex, and bone metastasis. Multivariate analysis showed that prior bevacizumab use ( $P=0.014 ; \mathrm{HR}, 1.79 ; 95 \% \mathrm{CI}, 1.12-2.86)$ and bone metastasis $(P=0.012$; HR, 2.44; 95\% CI, $1.21-4.92)$ were associated with PFS.

\section{Statistical analysis for OS after first-line treatment of all patients}

In the univariate analysis for OS after first-line treatment of all 190 patients, clinical factors with $P$-values $<0.2$ were PS $\geq 1$, unresectable primary tumors, liver metastasis, lung metastasis, and bone metastasis. Multivariate analysis showed that PS $\geq 1(P=0.0000027 ; \mathrm{HR}, 2.40 ; 95 \% \mathrm{CI}, 1.67-3.46)$ and lung metastasis $(P=0.00012$; HR, $0.54 ; 95 \% \mathrm{CI}, 0.39-0.74)$ were associated with OS after first-line treatment.

\section{Discussion}

We performed this study to determine whether the sequential use of cetuximab after bevacizumab failure decreases the efficacy of cetuximab in patients with mCRC. Among both the patients who underwent second- and third-line cetuximab treatments, PFS was significantly longer in those without prior bevacizumab treatment. This outcome indicates that sequential use of cetuximab after bevacizumab failure decreases the efficacy of cetuximab in patients with mCRC. 
Table 4 EGFR expressions by IHC of patients

\begin{tabular}{|c|c|c|c|c|}
\hline \multirow{3}{*}{$\begin{array}{l}\text { EGFR expressions, } \\
\text { n (\%) }\end{array}$} & \multicolumn{2}{|l|}{ Second line } & \multicolumn{2}{|l|}{ Third line } \\
\hline & $\begin{array}{l}\text { With prior } \\
\text { bevacizumab }\end{array}$ & $\begin{array}{l}\text { Without prior } \\
\text { bevacizumab }\end{array}$ & $\begin{array}{l}\text { With prior } \\
\text { bevacizumab }\end{array}$ & $\begin{array}{l}\text { Without prior } \\
\text { bevacizumab }\end{array}$ \\
\hline & $(n=2 I)$ & $(n=26)$ & $(n=109)$ & $(n=34)$ \\
\hline EGFR positive & $10(48)$ & $10(38)$ & $57(53)$ & $13(38)$ \\
\hline EGFR negative & I (5) & $2(8)$ & $5(5)$ & $0(0)$ \\
\hline Unknown & $10(48)$ & $14(54)$ & $47(43)$ & $21(62)$ \\
\hline
\end{tabular}

Abbreviations: EGFR, epidermal growth factor receptor; IHC, immunohistochemistry.

Various hypotheses of genetic instabilities in tumor cells have been proposed as mechanisms by which to decrease the efficacy of cetuximab by prior bevacizumab use. Some reports have shown that bevacizumab may promote EGFR resistance through VEGF overexpression.

Ellis found that at least part of the antitumor effect of cetuximab is mediated by inhibition of angiogenesis through interruption of upstream angiogenesis signaling pathways. ${ }^{4}$ Viloria-Petit et al examined the high-EGFRexpressing A431 cell line in mouse xenografts and reported that tumor cells resistant to anti-EGFR antibodies have exhibited VEGF production. ${ }^{5}$ Ciardiello et al investigated cetuximab resistance in GEO CRC cell lines induced by prolonged exposure to cetuximab in vivo. ${ }^{6}$ Cell lines derived from this work showed a dramatic increase in phosphorylated MAPK, increased COX-2 and VEGF protein expression levels, and increased secretion of VEGF. ${ }^{7}$
These studies indicate that VEGF overexpression promotes EGFR resistance.

Moreover, intravenous injection of bevacizumab reportedly leads to an increase in the plasma VEGF concentration in patients with cancer. High plasma VEGF levels after bevacizumab are possibly linked to VEGF overexpression in tumor cells and secondary promotion of EGFR resistance; bevacizumab may promote EGFR resistance through VEGF overexpression.

In a Phase I/II study, Willett et al showed that the plasma VEGF and placenta growth factor (PIGF) levels were high in patients with rectal cancer who received neoadjuvant bevacizumab in combination with chemoradiation therapy. ${ }^{7}$ They suggested that the plasma VEGF and PIGF levels may be useful as progressive disease biomarkers for anti-VEGF therapy. Weickhardt et al showed that a lower expression of VEGF-D was associated with a better OS and PFS in bevacizumab

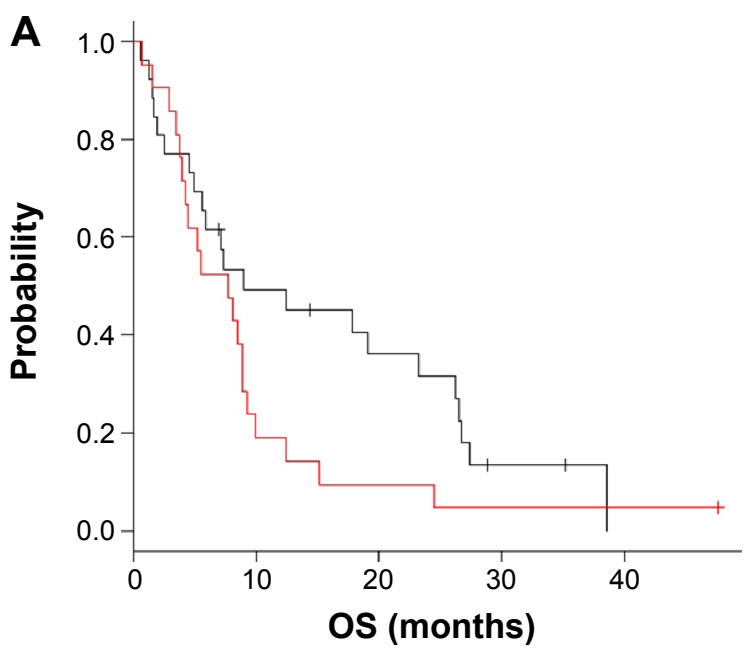

Number at risk

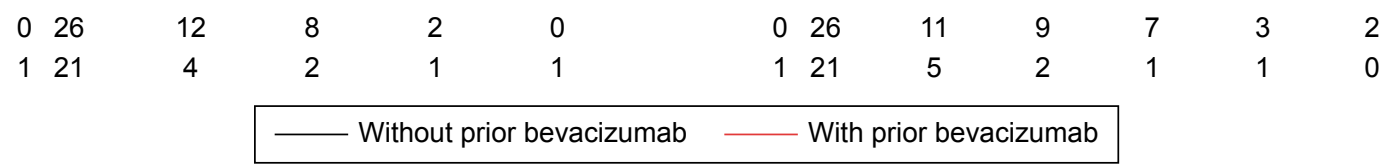

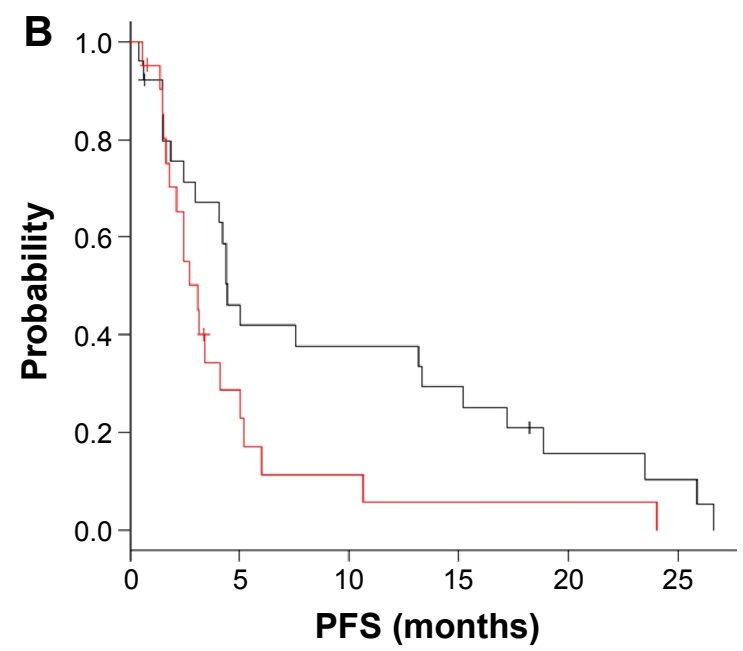

Number at risk

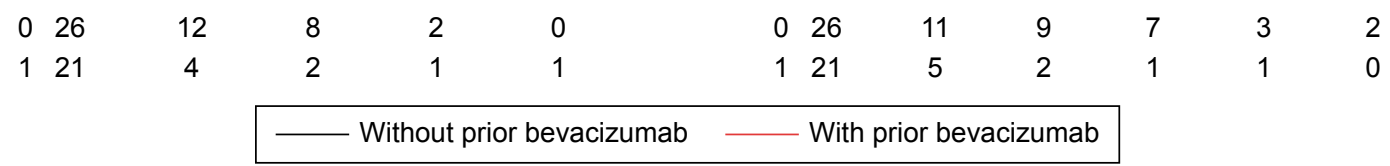

Figure 2 In the second-line cetuximab group, Kaplan-Meier curves of OS and PFS are illustrated stratified by with or without prior bevacizumab. Notes: (A) OS for patients receiving cetuximab as second line. (B) PFS for patients receiving cetuximab as second line.

Abbreviations: OS, overall survival; PFS, progression-free survival. 


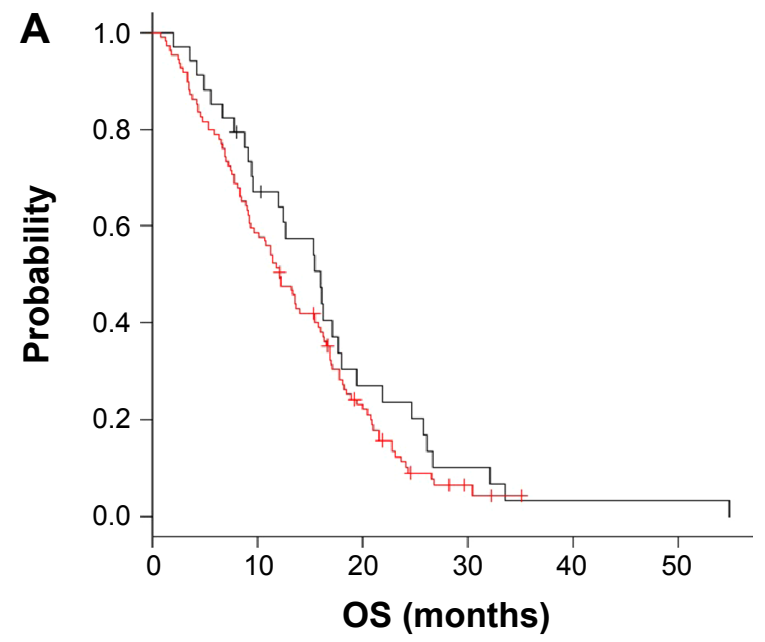

Number at risk

$\begin{array}{lllllll}0 & 34 & 22 & 8 & 3 & 1 & 1\end{array}$

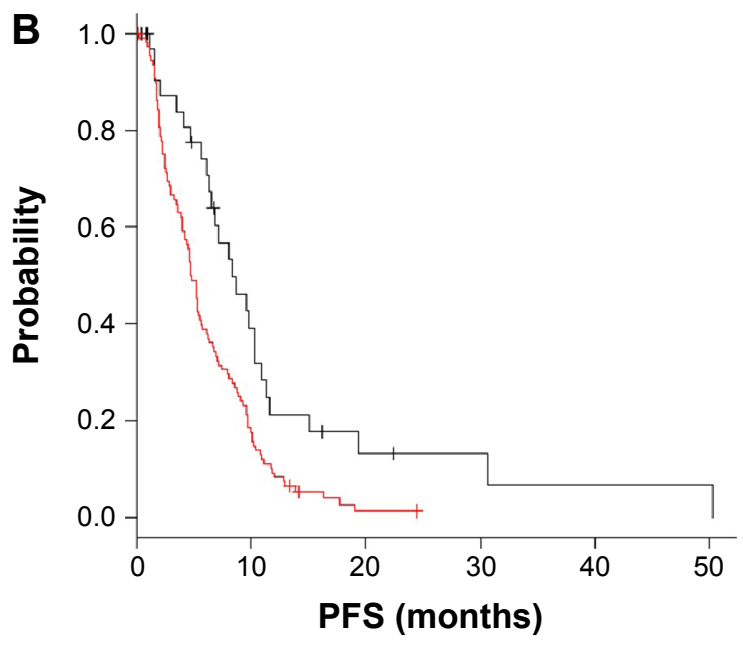

Number at risk

$\begin{array}{lllllll}0 & 34 & 11 & 3 & 2 & 1 & 1\end{array}$

$\begin{array}{lllllll}1 & 109 & 19 & 1 & 0 & 0 & 0\end{array}$

Without prior bevacizumab

With prior bevacizumab

Figure 3 In the third-line cetuximab group, Kaplan-Meier curves of OS and PFS are illustrated stratified by with or without prior bevacizumab.

Notes: (A) OS for patients receiving cetuximab as third line. (B) PFS for patients receiving cetuximab as third line.

Abbreviations: OS, overall survival; PFS, progression-free survival.

therapy and that higher expression of VEGF-D was predictive of bevacizumab resistance, most significantly with respect to PFS. ${ }^{8}$ Fan et al showed that cells chronically exposed to bevacizumab exhibited increased expression and activation of VEGF rexceptor-1 (VEGFR-1) and ligands for VEGFR-1, PIGF, and VEGF-B. These studies indicate the occurrence of VEGF overexpression after bevacizumab use. ${ }^{9}$

The decrease in the efficacy of cetuximab by prior bevacizumab use might be caused by the negative interaction between cetuximab and bevacizumab. The Phase III CAIRO2 trial showed that the addition of cetuximab to capecitabine, oxaliplatin, and bevacizumab resulted in significantly shorter PFS (9.6 months vs 10.7 months; $P=0.01$ ). ${ }^{10}$ The serum bevacizumab level is sustained for 3 months after the last bevacizumab infusion; cetuximab use within a short period of time after the last bevacizumab infusion might decrease the efficacy of cetuximab, similar to combination therapy comprising cetuximab and bevacizumab.

In our study, 89 (75\%) of 119 patients who underwent third-line cetuximab after prior bevacizumab therapy received cetuximab within 3 months from the last bevacizumab infusion. The median period from the last bevacizumab infusion was 2.6 months (range, 0.2-26.1 months). For supplemental analysis, we divided all the patients who had undergone third-line cetuximab therapy into three groups according to the duration of time from the last bevacizumab use: no prior bevacizumab infusion, $>3$ months from last bevacizumab infusion, and $<3$ months from last bevacizumab infusion. We estimated the OS and PFS using the Kaplan-Meier method. The median OS was 16.1 months in the no prior bevacizumab use group, 10.1 months in the $>3$ months from last bevacizumab infusion group, and 12.2 months in the $<3$ months from last bevacizumab infusion group ( $P=0.461$; Figure $4 \mathrm{~A}$ ). The median PFS was 8.4 months in the no prior bevacizumab use group, 5.7 months in the $>3$ months from last bevacizumab infusion group, and 4.8 months in the $<3$ months from last bevacizumab infusion group ( $P=0.0037$; Figure 4B). The supplemental analysis showed no significant difference in OS, but a shorter PFS especially in the $<3$ months from last bevacizumab infusion group; cetuximab use within 3 months after the last bevacizumab infusion decreased the efficacy of cetuximab.

In patients without prior bevacizumab, more patients had a PS $\geq 1$, which was associated with OS after first-line treatment. This bias may have influenced the lack of statistically significant differences in OS. Among the first six patients in the second-line treatment group, the OS of patients without prior bevacizumab treatment was inferior to that of patients with prior bevacizumab treatment. Actually, three of these six patients had a particularly worse baseline; they were aged $\geq 70$ years, had a poorer PS (1 or 2), and had lymphatic metastasis, all of which were associated with OS in the multivariate analysis.

The Phase III COMET trial of 108 patients with KRAS wild-type mCRC refractory to FOLFIRI + bevacizumab 

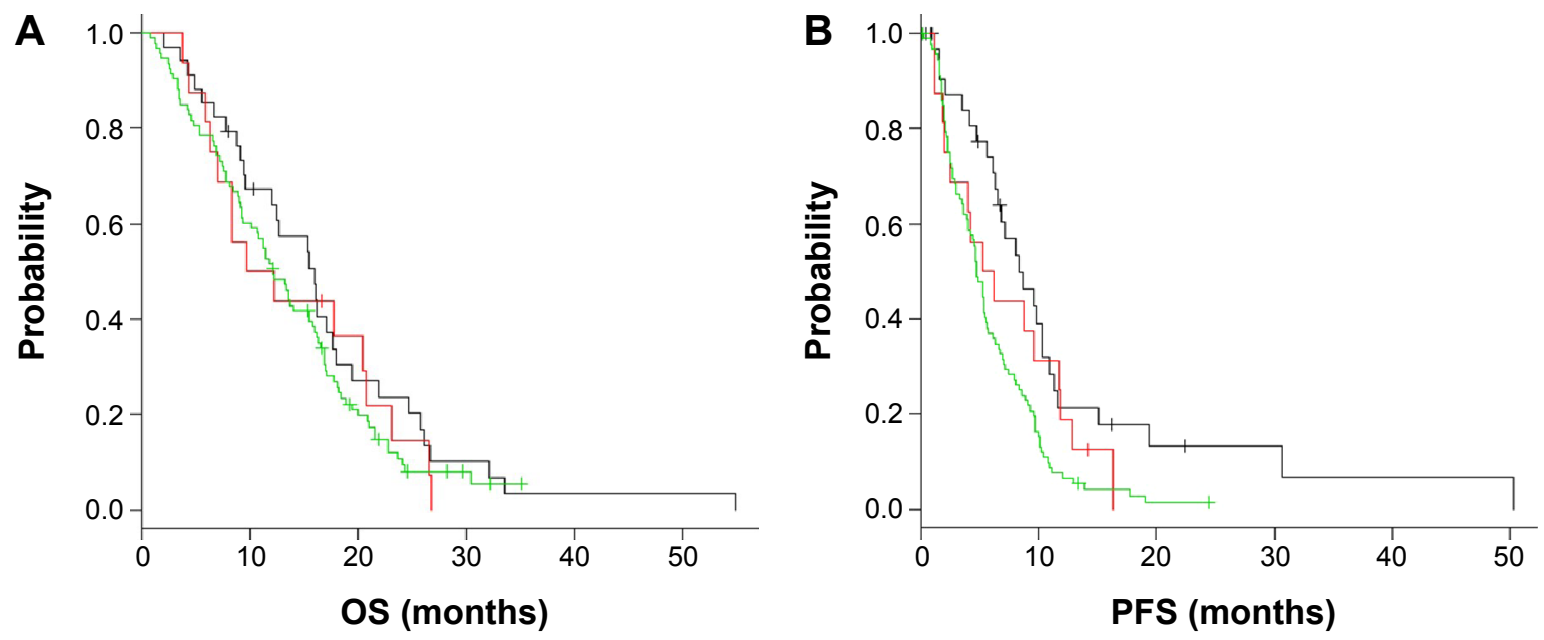

Number at risk

$\begin{array}{lllllll}0 & 34 & 22 & 8 & 3 & 1 & 1 \\ 1 & 16 & 8 & 5 & 0 & 0 & 0 \\ 2 & 93 & 56 & 16 & 3 & 0 & 0\end{array}$

Number at risk

$\begin{array}{lllllll}0 & 34 & 11 & 3 & 2 & 1 & 1 \\ 1 & 16 & 5 & 0 & 0 & 0 & 0 \\ 2 & 93 & 14 & 1 & 0 & 0 & 0\end{array}$

No prior bevacizumab —— $\geq 3$ months from last bevacizumab

$<3$ months from last bevacizumab

Figure 4 In the third-line cetuximab group, Kaplan-Meier curves of OS and PFS are illustrated stratified by the period from last bevacizumab; 0 : no prior bevacizumab, I: $>3$ months from last bevacizumab, and $2:<3$ months from last bevacizumab.

Notes: (A) OS for patients receiving cetuximab as third line. (B) PFS for patients receiving cetuximab as third line.

Abbreviations: OS, overall survival; PFS, progression-free survival.

compared two different sequences of second- and thirdline therapies, which were presented at the 2015 ASCO meeting by Cascinu et al: irinotecan + cetuximab followed by FOLFOX4 vs FOLFOX4 followed by irinotecan + cetuximab. Although the primary end point was not met (PFS was not statistically different), FOLFOX seems to be more effective than cetuximab in OS (18.6 months vs 12.4 months; HR, $0.79 ; P=0.28)$ as second-line treatment in patients receiving bevacizumab + FOLFIRI. This seems to confirm FIRE-3 trial and our study, suggesting that a prior anti-VEGF therapy may determine a lower sensitivity to a subsequent anti-EGFR treatment. ${ }^{11}$

From extended KRAS and NRAS testing in the FIRE-3 trial $^{12}$ and the PEAK study, ${ }^{2}$ patients with wild-type RAS tumors seemed to experience more clinical benefit with antiEGFR therapy. In our study, extended RAS testing could not be performed. Limited to patients without RAS mutation, decreasing efficacy of cetuximab after bevacizumab might be more emphasized.

\section{Limitations}

The limitations of this study include its retrospective design and the difference in patients' characteristics, especially imbalance of sex and age, which were not influenced outcomes in multivariate analysis, though, and the short follow-up time of 11.8 months and 13.7 months may not have allowed for adequate assessment of OS Accordingly, a long-term, double-blind trial should be performed using well-matched groups of patients to confirm our findings.

\section{Conclusion}

The present study showed that PFS was significantly longer in patients without prior bevacizumab treatment than in patients with prior bevacizumab treatment, indicating that prior bevacizumab treatment decreases the efficacy of cetuximab. Cetuximab may be more effective without prior bevacizumab treatment.

\section{Acknowledgments}

We thank Dr Izuma Nakayama, Dr Takeru Wakatsuki, Dr Mariko Ogura, Dr Masato Ozaka, and Dr Keisho Chin of the Department of Gastroenterology, Cancer Institute Hospital, Japanese Foundation for Cancer Research for their support and encouragement of this research. We thank medical writing services on behalf of Edanz Group Global Ltd.

\section{Author contributions}

All authors contributed toward data collection and statistical analysis, drafting and revising the manuscript and agree to be accountable for all aspects of the work. 


\section{Disclosure}

SM received commercial research grant from Taiho Pharmaceutical Co., Ltd. ES received honoraria from speakers bureau of Taiho Pharmaceutical Co., Ltd., Chugai Pharmaceutical Co., Ltd., Yakult Honsha Co., Ltd., Bristol-Myers Squibb, and Takeda Pharmaceutical Co., Ltd. NM received commercial research grant from Taiho Pharmaceutical Co., Ltd., Chugai Pharmaceutical Co., Ltd., Yakult Honsha Co., Ltd., Bristol-Myers Squibb, Takeda Pharmaceutical Co., Ltd., Merck Serono Co., Ltd., ONO Pharmaceutical Co., Ltd., and Bayer Yakuhin Co., Ltd. The authors have no other conflicts of interest in this work.

\section{References}

1. Heinemann V, von Weikersthal LF, Decker T, et al. FOLFIRI plus cetuximab versus FOLFIRI plus bevacizumab as first-line treatment for patients with metastatic colorectal cancer (FIRE-3): a randomised, open-label, phase 3 trial. Lancet Oncol. 2014;15(10):1065-1075.

2. Schwartzberg LS, Rivera F, Karthaus M, et al. A randomized, multicenter phase II study of panitumumab plus modified fluorouracil, leucovorin, and oxaliplatin (mFOLFOX6) or bevacizumab plus mFOLFOX6 in patients with previously untreated, unresectable, wild-type KRAS exon 2 metastatic colorectal cancer. J Clin Oncol. 2014;32(21): 2240-2247.

3. Norguet E, Dahan L, Gaudart J, Gasmi M, Ouafik L, Seitz JF. Cetuximab after bevacizumab in metastatic colorectal cancer: is it the best sequence? Dig Liver Dis. 2011;43(11):917-919.

4. Ellis LM. Epidermal growth factor receptor in tumor angiogenesis. Hematol Oncol Clin North Am. 2004;18(5):1007-1021.
5. Viloria-Petit A, Crombet T, Jothy S, et al. Acquired resistance to the antitumor effect of epidermal growth factor receptor-blocking antibodies in vivo: a role for altered tumor angiogenesis. Cancer Res. 2001;61(13): 5090-5101.

6. Ciardiello F, Bianco R, Caputo R, et al. Antitumor activity of ZD6474, a vascular endothelial growth factor receptor tyrosine kinase inhibitor, in human cancer cells with acquired resistance to antiepidermal growth factor receptor therapy. Clin Cancer Res. 2004;10(2):784-793.

7. Willett CG, Duda DG, di Tomaso E, et al. Efficacy, safety, and biomarkers of neoadjuvant bevacizumab, radiation therapy, and fluorouracil in rectal cancer: a multidisciplinary phase II study. J Clin Oncol. 2009; 27(18):3020-3026.

8. Weickhardt A, Williams D, Lee C, et al. Vascular endothelial growth factors (VEGF) and VEGF receptor expression as predictive biomarkers for benefit with bevacizumab in metastatic colorectal cancer (mCRC): analysis of the phase III MAX study. J Clin Oncol. 2011;29(suppl; abstr3531):228s.

9. Fan F, Samuel S, Gaur P, et al. Chronic exposure of colorectal cancer cells to bevacizumab promotes compensatory pathways that mediate tumour cell migration. Br J Cancer. 2011;104(8):1270-1277.

10. Tol J, Koopman M, Cats A, et al. Chemotherapy, bevacizumab, and cetuximab in metastatic colorectal cancer. N Engl J Med. 2009;360(6): 563-572.

11. Cascinu S, Rosati G, Nasti G, et al. A phase III multicenter trial comparing two different sequences of second/third line therapy (irinotecan/ cetuximab followed by FOLFOX-4 vs. FOLFOX-4 followed by irinotecan/cetuximab in K-RAS wt metastatic colorectal cancer (mCC) patients refractory to FOLFIRI/bevacizumab. J Clin Oncol. 2015; 33(suppl; abstr 3582).

12. Stintzing S, Jung A, Rossius L, et al. Analysis of KRAS/NRAS and BRAF mutations in FIRE-3: a randomized phase III study of FOLFIRI plus cetuximab or bevacizumab as first-line treatment for wild-type (WT) KRAS (exon 2) metastatic colorectal cancer (mCRC) patients. Eur J Cancer. 2013;49(suppl 3):S5-S6.
OncoTargets and Therapy

\section{Publish your work in this journal}

OncoTargets and Therapy is an international, peer-reviewed, open access journal focusing on the pathological basis of all cancers, potential targets for therapy and treatment protocols employed to improve the management of cancer patients. The journal also focuses on the impact of management programs and new therapeutic agents and protocols on
Dovepress

patient perspectives such as quality of life, adherence and satisfaction. The manuscript management system is completely online and includes a very quick and fair peer-review system, which is all easy to use. Visit http://www.dovepress.com/testimonials.php to read real quotes from published authors. 